\title{
Green Synthesis of Ag Nanoparticles from Aqueous Extracts of Leaves and Fruit of Casuarina equisetifolia against Candida albicans and other Clinical Isolates
}

\author{
Mahmoud Moustafa ${ }^{1,2^{*}}$, Ahmed Al-Emam ${ }^{3,4}$, Mahmoud Sayed ${ }^{5,6}$, Saad Alamri ${ }^{1,7}$, Huda Alghamdii ${ }^{1}$, Ali Shati ${ }^{1}$, \\ Sulaiman Alrumman ${ }^{1}$, Mohamed Al-Kahtani ${ }^{1}$, Eman Khalaf ${ }^{8}$, Thanaa Maghraby ${ }^{2}$ and Hanan Temerk ${ }^{2}$ \\ ${ }^{1}$ Department of Biology, College of Science, King Khalid University, 9004, Abha, Kingdom of Saudi Arabia \\ ${ }^{2}$ Department of Botany and Microbiology, Faculty of Science, South Valley University, Qena, Egypt \\ ${ }^{3}$ Department of Pathology, College of Medicine, King Khalid University, Abha, Saudi Arabia \\ ${ }^{4}$ Department of Forensic Medicine and Clinical Toxicology, Faculty of Medicine, Mansoura, University, Mansoura, Egypt \\ ${ }^{5}$ Physics Dep., Faculty of Science, King Khalid University, P. O. Box 9004, Abha, Saudi Arabia \\ ${ }^{6}$ Physics Department, Faculty of Science, Al-Azhar University, P. O. 71452, Assiut, Egypt \\ ${ }^{7}$ Prince Sultan Bin Abdulaziz Center for Environmental and Tourism Research and Studies-King Khalid University, Kingdom \\ of Saudi Arabia \\ ${ }^{8}$ Department of Microbiology and Immunology, Faculty of Pharmacy, Damanhour University, Damanhour, Egypt \\ *For correspondence: mfmostfa@kku.edu.sa; mfmostfa@kku.edu \\ Received 10 August 2020; Accepted 09 September 2020; Published 10 December 2020
}

\begin{abstract}
The prevalence of the infectious diseases caused by Candida species and other human pathogenic microbes has led to the discovery of some natural agents against multidrug resistant microbes. Therefore, this research was designated to determine the capability of synthesized nanoparticles (NPs) from aqueous extract of leaves and fruits of Casuarina equisetifolia as anticandidal and antibacterial against some microbes. Silver NPs (AgNPs) were successfully gained from aqueous leaves and fruit extracts of $C$. equisetifolia which is defined on the basis of UV-visible spectroscopy, X-ray diffraction analysis and scanning electron microscope. The results showed that leaves and fruits extracts of $C$. equisetifolia acts as an excellent capping agent. XRD based on the FWHM analysis showed that $\mathrm{AgCl}$ and $\mathrm{Ag}$ had an average NPs size of $90.97 \mathrm{~nm}$ and $71.28 \mathrm{~nm}$, respectively for fruits and $15.33 \mathrm{~nm}$ and $14.01 \mathrm{~nm}$, respectively for leaves. UV-visible spectroscopy showed a maximum absorbance at $442 \mathrm{~nm}$ for fruits and $433 \mathrm{~nm}$ for leaves. SEM showed that the size of NPs from leaves lied in between of (30 to180 nm) and for fruits (70 to $250 \mathrm{~nm}$ ). Candida albicans was severely affected by NPs of leaves with inhibition zone (3.03 \pm $1.61 \mathrm{~cm})$ and NPs of fruits had $(1.37 \pm 0.15 \mathrm{~cm})$ inhibition zone. Nanoparticles from leaves exhibited maximum activity against $P$. mirabilis $(3.52 \pm 0.13 \mathrm{~cm})$ and low activities against $M$. luteus $(1.50 \pm 0.18 \mathrm{~cm})$ inhibition zones. In conclusion, these eco-friendly synthesized AgNPs from leaves and fruits of $C$. equisetifolia could be used as competitive alternative natural drugs than conventional synthetic chemicals. (C) 2021 Friends Science Publishers
\end{abstract}

Keywords: Casuarina equisetifolia; AgNPs; Anticandidal; Antibacterial; Aqueous extracts

\section{Introduction}

The construction of functional molecule at micro level is of great interest for nanotechnology techniques. Nanomaterials (NPS) are used extensively in the area of medicine for the purpose of drug delivery, diagnosis, treatment of cardiovascular disorders, wound healing and production of antimicrobial agents. They exhibit unique physico-chemical properties, which are not observed in either single molecules or bulk metals itself (Dauthal and Mukhopadhyay 2016). They are used for multiple purposes for industrial and in medicinal applications
(Thakkar et al. 2010; Linic et al. 2015). Silver nanoparticles (AgNPs) were more concerned than other metallic nanoparticles (MNPs) because of their special properties such as magnetic, optical polarization, electrical conductivity (EC) and prominent antimicrobial activity (Evanoff and Chumanov 2005). Considering the presence of various metals in nature, only some of them, such as silver, gold, palladium and platinum, are extensively synthesized in nanostructured form (Yoon et al. 2010; Yang et al. 2017). Three different methods of synthesis for NPs developed are chemical, physical and green synthesis (Oves et al. 2018; Saratale et al. 2018).

To cite this paper: Moustafa M, A Al-Emam, M Sayed, S Alamri, H Alghamdii, A Shati, S Alrumman, M Al-Kahtani, E Khalaf, T Maghraby, H Temerk (2021). Green synthesis of ag nanoparticles from aqueous extracts of leaves and fruit of Casuarina equisetifolia against Candida albicans and other clinical isolates. Intl J Agric Biol 25:117-122 
Physical methods require expensive equipment, high temperature and high pressure. For the synthesis of nanoparticles using chemical processes, harmful substances are used, which can cause significant harm to the atmosphere and to living organisms. Due to these disadvantages, the use of chemical and physical methods is limited and gradually is replaced by green synthesis, which is a more environmentally friendly and cheaper method. Green synthesis in all process is similar to chemical reduction where costly chemical reducing agent is substituted by a natural product extract, such as leaves, root or fruits are applied for NPs synthesis of metal or metal oxide (Hussain et al. 2016). Natural product NPs are considered to be environmental friendly and safe (Jayaseelan et al. 2012; Gopinath et al. 2014), free from environmental pollution (Chandran et al. 2006; Huang et al. 2007), cheaper (Mittal et al. 2013) and ideal for mass manufacturing (Iravani 2011). In addition, biologic development of NPs makes it possible to recycle expensive metal salts such as silver and gold contained in waste streams (Wang et al. 2009).

Plants, bacteria, fungi, algae, etc. are commonly used for green nanoparticle synthesis (Chen et al. 2015; Khan et al. 2016; Agarwal et al. 2017). The ability of pathogenic candida and bacteria to resist against various drugs is a major obstacle in medical practice that restricts the effectiveness of such normal medications (Quelemes et al. 2013). Such disadvantages give researchers enormous opportunities to create new substances, such as AgNPs, to counter such problem.

Casuarina equisetifolia $\mathrm{L}$. is predominantly a monoecious species belonging to the Casuarinaceae family (Binggeli et al. 1999). It is planted as ornamental in the street side or as a fence around house or may be growing wildly in abandoned area. It is found in many climatic regime, usually grow near sea level but succeeding under cultivation at heights up to 1,400 meters and can grow under saline environments (Jensen 1995; Garrity et al. 2006). The plant contains several phytochemical components such as glycosides, quercetin, triterpenoids, tannins, rutin and casuarine, catechol derivatives etc. (Ramanathan et al. 2012). The existence of natural secondary metabolites shows the tree species as important source for use as an astringent, diuretic agent for coughing, diarrhea, beri-beri, colic and as toothache. Biological properties such as hypoglycemic anticancer activity of this tree species have been characterized (Han 1998; Parekh et al. 2006).

Candidiasis is caused by various Candida species and considered as one of the most prevalent fungal infections to humans, especially by $C$. albicans. There are no reports till date about the biosynthesis of (AgNPs) by applying aqueous extract of leaf and fruit of the Casuarinaceae family. Herein, an effort has been made to examine NPs from leaf and fruit extract of the $C$. equisetifolia against $C$. albicans isolates and against a range of human pathogenic microbes as potential drugs for pharmaceutical application. Also, in the present study NPs from leaf and fruits had been characterized using various techniques. This research was based on a rather simple hypothesis that plant aqueous extracts contain a group of polar compounds rather than secondary metabolites. Thus, depending on that fact, simple preparation of AgNPs from leaves and fruits of $C$. equisetifolia have been relaying on water extraction.

\section{Materials and Methods}

\section{Collection of plant materials}

Leaves and fruits samples of $C$. equisetifolia were collected from Abha region, KSA during the month of May in 2019 (Latitude: 18.2114609; Longitude: 42.4999752). The obtained materials were thoroughly rinsed in flowing tap water to remove adhesive particles, and then with distilled water until no traces of foreign material remained.

\section{Preparation of plant extract}

A $30 \mathrm{~g}$ of fresh leaves and fruits of $C$. equisetifolia was crushed thoroughly with a blender (Philips, Germany) to a homogeneous mass with the addition of $30 \mathrm{~mL}$ of DW (1 $\mathrm{g} / \mathrm{mL}$ ). Then extract was filtered using Whatman No 1 filter paper, and the supernatant was collected and stored at $4{ }^{\circ} \mathrm{C}$ for further analysis.

\section{Synthesis of silver nanoparticle}

Silver nitrate reagent $(0.5 \mathrm{mM})$ was made using deionized water, to synthesis silver nanoparticle from leaves and fruits extract of $C$. equisetifolia. Ten $\mathrm{mL}$ from each was added to

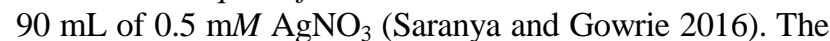
mixture was heated at $70^{\circ} \mathrm{C}$ for $3 \mathrm{~min}$. The reduction of aqueous silver ions by both extract to form stable AgNPs was noted with the formation of yellow, brown and then blackish colored solution (Kirthika et al. 2014).

\section{Characterization of silver nanoparticles}

UV-Vis Spectrophotometer: Reduction of $\mathrm{Ag}^{+}$ions was spectrophometrically monitored using double-beam UV-Vis spectroscopy (Hitachi, U-3010) by dissolving a small aliquot of the reaction solution into distilled water. The wavelength of 350-500 nm was measured and the peak was recorded.

Powder X-ray Diffraction (XRD): Powdered silver nanoparticles were mounted on a microscopic slide and air dried at $24^{\circ} \mathrm{C}$ overnight. The crystalline form, phase detection and grain size using XRD (Shimadzu, 6000 Diffractometer, Japan) were achieved.

Scanning electron microscope (SEM): The AgNPs morphology images from leaves and fruits extracts from $C$. equisetifolia were studied via SEM (JSM-7500 F; JOELJapan). Some drops from the sample colloidal solution were dried at $60^{\circ} \mathrm{C}$ in a high stability furnace on microscopic sheet and then observed. 


\section{Anticandidal and antibacterial activities}

In vitro antimicrobial behavior of processed silver nanoparticles using leaves and fruit extracts of $C$. equisetifolia was investigated. Candida albicans and other six human pathogens viz., Proteus mirabilis, Psedomonas aeruginosa, Staphylococcus aureus, Shigella flexneri, Klebsiella pneumoniae and Micrococcus luteus were used for anticandidal and antibacterial studies, which were obtained from the Department of Microbiology, King Khalid University, Saudi Arabia. These microbes were grown in nutrient broth for $48 \mathrm{~h}$. Agar well diffusion method was used to screen the antibacterial and anticandidal activities of NPs gained from aqueous extract of leaves and fruits of C. equisetifolia (Daoud et al. 2019). Potato Dextrose Agar and nutrient agar media were prepared for $C$. albicans and for bacterial strains respectively and from each $20 \mathrm{~mL}$ were poured into sterilized Petri dish. Upon solidification, $1 \mathrm{~mL}$ from fresh candida and bacterial culture was pipetted in the center of sterile Petri dish and swapped uniformly upon the surface of solid media using Sterile Cotton swabs. In each plate two wells were punctured by autoclaved cork borer $(6 \mathrm{~mm}$ in diameter) into agar plates containing inoculums. Then $100 \mu \mathrm{L} \mathrm{AgNPs} \mathrm{of} \mathrm{each} \mathrm{extract}$ was applied to the respective well. Plates were refrigerated for $30 \mathrm{~min}$ to allow the extracts to spread well into the agar. The plates were then incubated at $37^{\circ} \mathrm{C}$ for $48 \mathrm{~h}$. Anticandidal and antibacterial activities were detected by measuring the inhibition zone (including the well diameter) that occurred during the incubation period. DMSO at a concentration of $10 \%$ was employed as a negative control and Cefoxitin ( $30 \mathrm{mcg}$ ) disc was used as positive control. Each plate was examined after incubation and the diameters of the inhibition zone were determined using the millimeter ruler. A region of inhibition of about $9 \mathrm{~mm}$ or more around the holes was defined that the extracts have antimicrobial substance (Alamri and Moustafa 2012). All experiment was performed in three replicates.

\section{Statistical analysis}

Results were subjected to one-way analysis of variance (ANOVA) followed by Least Significant Difference (LSD) Post Hoc test of three replicates \pm standard deviation (SD). Differences between means in relation to positive control were considered significant at $p$-value $<0.05$.

\section{Results}

\section{Antimicrobial activity}

The antimicrobial activity behavior of the biosynthesized AgNPs is shown in Fig. 1. There was remarkable inhibition activity of AgNPs against C. albicans, Gram-positive and Gram-negative bacteria. The maximum inhibitory effects of AgNPs were observed against all tested microbial strains

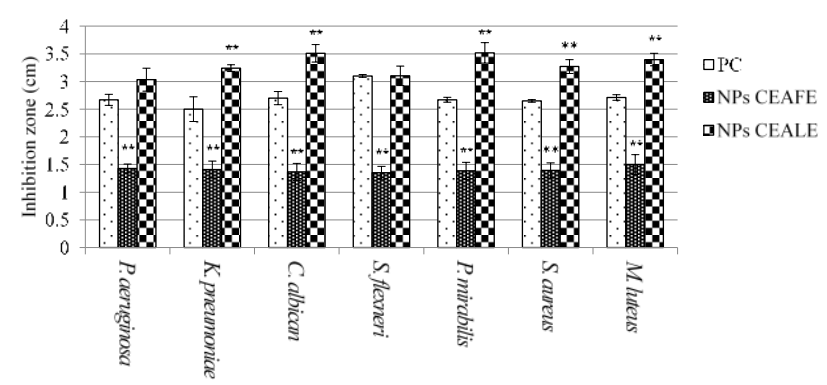

Fig. 1: AgNPs anticandidal and antibacterial activities from leaves and fruits of $C$. equisetifolia aqueous extracts against $C$. albican, $P$. aeruginosa, $K$. pneumoniae, $S$. flexneri, $P$. mirabilis, $S$. aureus and $M$. luteus. PC, positive control. Significant differences $(* P \leq$ $0.05 ; * * P \leq 0.01$ ), between treatments in relation to $\mathrm{PC} \pm \mathrm{SD}$ of the mean for $\mathrm{n}=3$

when prepared from $C$. equisetifolia leaf extract. While AgNPs from fruits extract of $C$. equisetifolia showed low antimicrobial activities against all tested microbial strains. For C. albicans the zone of inhibition from AgNPs of aqueous fruits was $(1.37 \pm 0.15 \mathrm{~cm})$. With an inhibition zone between $1.5-1.35 \mathrm{~cm}$, it was considered that all the tested strains were susceptible to AgNPs from fruits extract of $C$. equisetifolia, which implied that the extract have antimicrobial properties (Nascimento et al. 2000; Alamri and Moustafa 2012). NPs of aqueous fruits extract showed maximum antibacterial activities against $M$. luteus, followed by $P$. aeruginosa and moderate activity against $S$. aureus, $P$. mirabilis, K. pneumoniae and S. flexneri (Fig. 1). AgNPs from aqueous leaves extract of $C$. equisetifolia showed potent antimicrobial activities against all tested microbial strains more than fruits extract between 61.06 and $52.97 \%$. C. albicans was severely affected by AgNPs leaves extract with a zone of inhibition of $3.03 \pm 1.61 \mathrm{~cm}$. AgNPs of aqueous leaves showed maximum antibacterial activities against $P$. mirabilis $(3.52 \pm 0.13 \mathrm{~cm})$, followed by $K$. pneumoniae $(3.24 \pm 0.06 \mathrm{~cm})$ and M. luteus $(3.40 \pm 0.14 \mathrm{~cm})$. $S$. flexneri, $P$. aeruginosa and $S$. aureus demonstrated susceptibility in the range between $(3.27 \pm 0.11 \mathrm{~cm}$ and $3.03 \pm 0.21 \mathrm{~cm}$ with AgNPs from leaves extract. The percentage susceptibility differences among various bacterial strains between AgNPs of leaves and fruits extracts was; $P$. mirabilis $>$ S. aureus $>$ S. flexneri $>$ K. pneumoniae $>M$. luteus $>P$. aeruginosa. C. albican was very sensitive to $\mathrm{AgNP}_{\mathrm{S}}$ from leaves and fruits extract as the maximum differences between them was found to be $61.06 \%$. DMSO showed no antimicrobial activities against all tested microbes while positive control inhibited microbial growth in the range between 3.10 and $2.55 \mathrm{~cm}$ increased as compared to AgNPs of fruits extract which was 3.1 to 1.93\%. Efficacy of AgNPs from leaves extract was higher than positive control by $39.96 \%$ against $P$. mirabilis to $15.48 \%$ against $P$. aeruginosa. Remarkable differences were also found against $C$. albicans by AgNPs of leaves extract of C. equisetifolia being more than positive control (Fig. 1). 


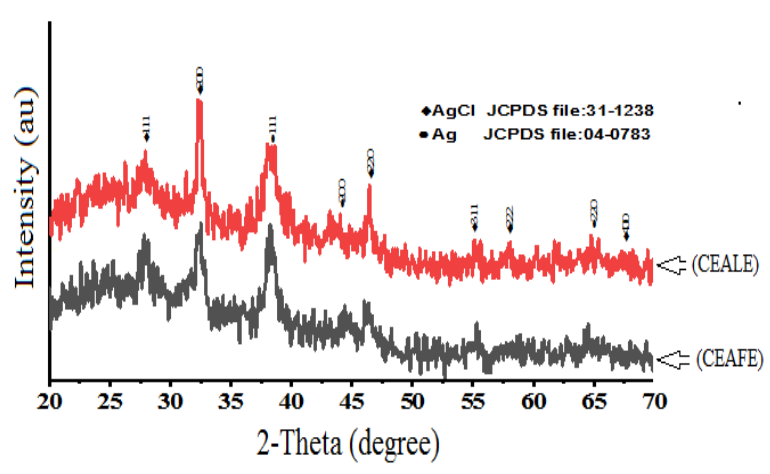

Fig. 2: X-ray diffraction pattern of AgNPs from C. equisetifolia aqueous leaves extract (CEALE) and aqueous fruits extract (CEAFE)

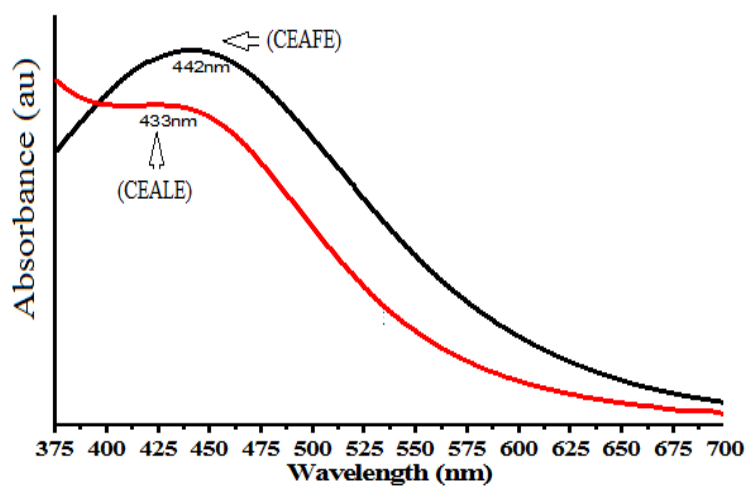

Fig. 3: The UV/Vis absorption spectra of AgNPs from $C$. equisetifolia aqueous leaves extract (CEALE) and aqueous fruits extract (CEAFE)

\section{NPs characterizations using XRD}

Fig. 2 shows the XRD pattern of NPs from C. equisetifolia leaves aqueous extract (CELAE) and fruits aqueous extract (CEAFE). There was similarity to some extent between the positions of the diffraction peaks for both patterns. For CEAFE, the diffraction peaks were found at 2-theta values at $38.20^{\circ}$ (111), $44.40^{\circ}$ (200), and $64.55^{\circ}$ (220), corresponded to the crystal planes of the face-centered cubic(fcc) Ag (JCPDS card No. 04-0783); whilst, five additional diffraction peaks appearing at 2-theta values of $27.78^{\circ}$ (111), $32.49^{\circ}$ (200), $46.37^{\circ}$ (220), $54.47^{\circ}$ (311), $57.31^{\circ}$ (222) and $67.10^{\circ}$ (400) correspond to the crystal planes of the face-centered cubic (fcc) $\mathrm{AgCl}$ (JCPDS file:31-1238). Similarly, for CELAE extraction, the diffraction peaks at 2 -theta values at $38.08^{\circ}$ (111), $43.93^{\circ}$ (200), and $64.74^{\circ}(220)$, correspond to the crystal planes of the face-centered cubic(fcc) Ag (JCPDS card No. 04-0783); but, five additional diffraction peaks appearing at 2-theta values of $27.81^{\circ}(111), 32.37^{\circ}(200), 46.41^{\circ}(220), 55.10^{\circ}$ (311), $57.96^{\circ}(222)$ and $67.22^{\circ}$ (400) correspond to the crystal planes of the face-centered cubic(fcc) $\mathrm{AgCl}$ (JCPDS file:31-1238). These data confirmed that $\mathrm{Ag} / \mathrm{AgCl}$ nanoparticles are synthesized via both extraction of plant. Other peaks occured in the XRD pattern that could be due to the crystallization of bio-organic particles on the surface of the AgNPs. Furthermore, the average crystal size (Table 1) was determined by the Debye-Scherrer formula: $D=(0.9$ $\lambda) /(\beta \cos \theta)$, where $\mathrm{D}$ is the average size of the crystals, $\lambda$ is the wavelength of radiation, $\beta$ is the full width at half the maximum height (FWHM) and $\theta$ is the position of the maximum diffraction peak. Based on the FWHM of the diffraction peak (200) of the $\mathrm{AgCl}$ crystals and (111) the diffraction peak of the $\mathrm{Ag}$ crystals, the $\mathrm{AgCl}$ and $\mathrm{Ag}$ average nanoparticle size was determined and found as 90.97 and $71.28 \mathrm{~nm}$, respectively for CEAFE, whereas D was reduced to 15.33 and $14.01 \mathrm{~nm}$ for CELAE.

\section{UV-vis spectrophotometer and SEM analysis}

In the present study, the solution of $\mathrm{AgNO}_{3}$ was immediately turned into blackish after the addition of $C$. equisetifolia leaves and fruit extract as reducing agents in all samples showing the formation of AgNPs. Fig. 3 shows the typical UV spectra of the reaction solution with a localized surface plasmon resonance (SPR) of about 442 and $433 \mathrm{~nm}$ for AgNPs synthesized using extract of (CEAFE) and (CEALE), respectively, which strongly indicated the formation of AgNPs. Moreover, there was SPR semi-flat peak, which resulted from the presence of $\mathrm{Ag} / \mathrm{AgCl} \mathrm{NPs}$.

SEM images of the synthesized AgNPs extracted by CEAFE and CEALE respectively are shown in Fig. 4. The CEALE AgNPs showed ultrafine rounded to sub-rounded nano-particles of average size $30-180 \mathrm{~nm}$ dispersed on the surface of bio-organic sheets as a residue material. However, CEAFE AgNPs exhibited different particlesmorphologies and the particle size was between $70-250 \mathrm{~nm}$ relatively more than those of CEALE.

\section{Discussion}

Based on gained NPs antimicrobial analyses against tested microbes, it was noticed that the mode of action included bactericidal effect against bacterial strains and candidcidal against $C$. albicans of AgNPs were most possibly due to the binding of AgNPs to the cell wall and the production of free radicals (Pirtarighat et al. 2019). Our explanation why NPs prepared from leaves more potent than fruits this probably due to leaves have more chemicals than fruits. In addition, it was reported that AgNPs could disrupt the permeability of the membrane by entering the cell membrane and inducing intracellular ATP leakage and finally causing the cell death (Hajipour et al. 2012; Ajitha et al. 2014). Positively charged ions from $\mathrm{Ag}^{+}$seem to have a strong tendency to act with phosphorus and sulfur in biomolecules such as DNA and RNA, so that such attachment of small NPs from $C$. equisetifolia leaves leads to the interruption of the roles of DNA and RNA in the cell (Hajipour et al. 2012). 
Table 1: Experimental diffraction angles, FHWM, d-spacing, diffraction planes and particles size of silver (Ag) and silver chloride $(\mathrm{AgCl})$ extracted by aqueous extracts from fruits (CEAFE) and leaves (CEALE) of $C$. equisetifolia

\begin{tabular}{|c|c|c|c|c|c|c|c|}
\hline Sample & Crystalline phases & Diffraction angle (degree) & FHWM in radians & Lambda (nm) & d-spacing (nm) & Diffraction plane & Particle size (nm) \\
\hline \multirow[t]{2}{*}{ CEAFE } & $\mathrm{AgCl}$ & 38.20 & 0.001587 & 0.154 & 0.275 & $(200)$ & 90.97 \\
\hline & $\mathrm{Ag}$ & 32.49 & 0.002058 & - & 0.235 & (111) & 71.28 \\
\hline CEALE & $\mathrm{AgCl}$ & 32.37 & 0.00942 & 0.154 & 0.276 & $(200)$ & 15.33 \\
\hline
\end{tabular}

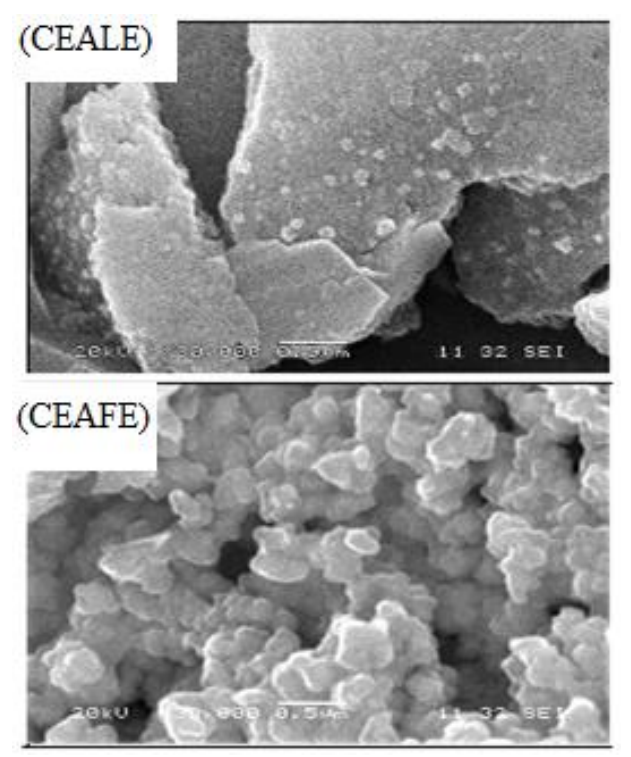

Fig. 4: SEM of AgNPs from C. equisetifolia aqueous leaves extract (CEALE) and aqueous fruits extract (CEAFE)

It has been reported that the smaller the particle size of the AgNPs, the more microbial cell damage will occur, due to oxidative stress response due to reactive oxygen species (ROS). Again, ROS caused DNA damage, and the rupture of the cell membrane of the microbial strains. In agreement with our findings that nanoparticles have a greater surface area available for contact that improves the bactericidal and candidcidal effect than large particles (Raffi et al. 2008). Previous works showed that various NPs from plant extracts had promising candidcidal effect as $\mathrm{Zn}$ nanoparticles combined with fluconazole could inhibit the vaginal candidiasis (Nazari 2020).

As the particles size increase, the position of the absorption peak is shifted to the long wave, and the larger the particle size, the more visible the phenomenon of red transformation. By altering extraction using $C$. equisetifolia aqueous leaves extract to aqueous fruits resulting in a blue shift from the SPR range from 442 to $434 \mathrm{~nm}$ implied that smaller NPs were found there. Small AgNPs may be due to higher nucleation rate with the improved reduction process which supports more potent antimicrobial activities (Khan et al. 2013; Dong et al. 2019).

Agglomeration of AgNPs in the form of nanoclusters may be due to the effect of plant extract during sample preparation for SEM. Again, this supports the view that tiny size of nanoparticles may assist in easy penetration of microbial cell wall and destroy various organelles. Biosynthesized small NPs have been identified as being able to inhibit/destroy microbial cells (Singh et al. 2019). In agreement with previous experimental results (Saranya and Gowrie 2016), this study suggested that the application of AgNPs synthesized from leaves and fruits aqueous extract of $C$. equisetifolia, could be used as an effective antimicrobial agent.

\section{Conclusion}

Using aqueous extract of $C$. equisetifolia yielded stable AgNPs as confirmed by UV-Vis, XRD and SEM techniques. Overall, AgNPs from aqueous leaves and fruits extracts from $C$. equisetifolia possessed antibacterial and anticandidal activities as they could inhibit the growth of selected $C$. albicans and bacterial strains. AgNPs from leaves of $C$. equisetifolia had more potent antimicrobial activity than fruits which may be used as natural agent to control the growth of many pathogenic microbes. More research is needed to characterize NPs from $C$. equisetifolia in order to find better antimicrobial activity.

\section{Acknowledgments}

The authors thank the Deanship of Scientific Research at King Khalid University for funding this work through Grant No. R.G.P.1/134/40

\section{Author Contributions}

MM, MS, EK, TM and HT planned and doing the experimental works, AA and HA interpreted the results and MM, MS, MA, EK, TM, SA, SUA, AS made the write up, analyzed the data and edited the review.

\section{References}

Agarwal H, SV Kumar, S Rajeshkumar (2017). A review on green synthesis of zinc oxide nanoparticles-an eco-friendly approach. Res-Efficient Technol 3:406-413

Ajitha B, YAK Reddy, PS Reddy (2014). Biogenic nano-scale silver particles by Tephrosia purpurea leaf extract and their inborn antimicrobial activity. Spectrochim Acta A 121:164-172

Alamri SA, MF Moustafa (2012). Antimicrobial properties of 3 medicinal plants from saudi arabia against some clinical isolates of bacteria. Saudi Med J 33:272-277

Binggeli P, JB Hall, JR Healey (1999). Invasive woody plants. In: The Overstorey Book: Cultivating Connections with Trees, pp:383-388. $2^{\text {nd }}$ edn. Elevitch CR (Ed.). Permanent Agricultural Resources Publisher, Holualoa, Hawaii, USA 
Chandran SP, M Chaudhary, R Pasricha, A Ahmad, M Sastry (2006). Synthesis of gold nanotriangles and silver nanoparticles using Aloe vera plant extract. Biotechnol Prog 22:577-583

Chen J, X Liu, C Wang, SS Yin, XL Li, WJ Hu, M Simon, ZJ Shen, Q Xiao, CC Chu (2015). Nitric oxide ameliorates zinc oxide nanoparticles-induced phytotoxicity in rice seedlings. $J$ Hazard Mater 297:173-182

Daoud A, D Malika, S Bakari, N Hfaiedh, K Mnafgui, A Kadri, N Gharsallah (2019). Assessment of polyphenol composition, antioxidant and antimicrobial properties of various extracts of date palm pollen (dpp) from two tunisian cultivars. Arab $J$ Chem 12:3075-3086

Dauthal P, M Mukhopadhyay (2016). Noble metal nanoparticles: Plantmediated synthesis, mechanistic aspects of synthesis, and applications. Ind Eng Chem Res 55:9557-9577

Dong Y, H Zhu, Y Shen, W Zhang, L Zhang (2019). Antibacterial activity of silver nanoparticles of different particle size against Vibrio natriegens. PLoS One 14; Article e 0222322

Evanoff Jr DD, G Chumanov (2005). Synthesis and optical properties of silver nanoparticles and arrays. Chem Phys Chem 6:1221-1231

Garrity DP, A Okono, M Grayson, S Parrott (2006). World Agroforestry into the Future. World Agroforestry Centre, ICRAF Nairobi, Kenya

Gopinath K, V Karthika, S Gowri, V Senthilkumar, S Kumaresan, A Arumugam (2014). Antibacterial activity of ruthenium nanoparticles synthesized using Gloriosa superba 1. Leaf extract. J Nanostructure Chem 4; Article 83

Hajipour MJ, KM Fromm, AA Ashkarran, DJ de Aberasturi, IR de Larramendi, T Rojo, V Serpooshan, WJ Parak, M Mahmoudi (2012). Antibacterial properties of nanoparticles. Trends Biotechnol 30:499-511

Han S (1998). Medicinal plants in the south pacific. World Health Organization (WHO) Regional Publications. Western Pac Ser 19:7-8

Huang J, Q Li, D Sun, Y Lu, Y Su, X Yang, H Wang, Y Wang, W Shao, N $\mathrm{He}$ (2007). Biosynthesis of silver and gold nanoparticles by novel sundried Cinnamomum camphora leaf. Nanotechnology 18; Article 105104

Hussain I, N Singh, A Singh, H Singh, S Singh (2016). Green synthesis of nanoparticles and its potential application. Biotechnol Lett 38:545-560

Iravani S (2011). Green synthesis of metal nanoparticles using plants. Green Chem 13:2638-2650

Jayaseelan C, AA Rahuman, AV Kirthi, S Marimuthu, T Santhoshkumar, A Bagavan, K Gaurav, L Karthik, KB Rao (2012). Novel microbial route to synthesize $\mathrm{ZnO}$ nanoparticles using aeromonas hydrophila and their activity against pathogenic bacteria and fungi. Spectrochim Acta A 90:78-84

Jensen M (1995). Trees Commonly Cultivated in Southeast Asia: An Illustrated Field Guide. Craftsman Press Co., Ltd., Bangkok, Thailand

Khan M, M Khan, SF Adil ,MN Tahir, W Tremel, HZ Alkhathlan, A AlWarthan, MRH Siddiqui (2013). Green synthesis of silver nanoparticles mediated by Pulicaria glutinosa extract. Intl $J$ Nanomed 8; Article 1507

Khan ST, J Musarrat, AA Al-Khedhairy (2016). Countering drug resistance, infectious diseases, and sepsis using metal and metal oxides nanoparticles: Current status. Colloids Surf $B$ Biointerfaces 146:70-83

Kirthika P, B Dheeba, R Sivakumar, SS Abdulla (2014). Plant mediated synthesis and characterization of silver nanoparticles. Intl $J$ Pharm Pharm Sci 6:304-310
Linic S, U Aslam, C Boerigter, M Morabito (2015). Photochemical transformations on plasmonic metal nanoparticles. Nat Mater 14:567-576

Mittal AK, Y Chisti, UC Banerjee (2013). Synthesis of metallic nanoparticles using plant extracts. Biotechnol Adv 31:346-356

Nascimento GG, J Locatelli, PC Freitas, GL Silva (2000). Antibacterial activity of plant extracts and phytochemicals on antibiotic-resistant bacteria. Braz J Microbiol 31:247-256

Nazari R (2020). Synergistic antifungal effect of fluconazole combined with $\mathrm{ZnO}$ nanoparticles against candida albicans strains from Vaginal candidiasis. Adv Mater 14:26-32

Oves M, M Aslam, MA Rauf, S Qayyum, HA Qari, MS Khan, MZ Alam, S Tabrez, A Pugazhendhi, IM Ismail (2018). Antimicrobial and anticancer activities of silver nanoparticles synthesized from the root hair extract of Phoenix dactylifera. Mater Sci Eng C 89:429-443

Parekh J, D Jadeja, S Chanda (2006). Efficacy of aqueous and methanol extracts of some medicinal plants for potential antibacterial activity. Turk J Biol 29:203-210

Pirtarighat S, M Ghannadnia, S Baghshahi (2019). Green synthesis of silver nanoparticles using the plant extract of Salvia spinosa grown in vitro and their antibacterial activity assessment. J Nanostr Chem 9:1-9

Quelemes PV, FB Araruna, BE De Faria, SA Kuckelhaus, DA Da Silva, RZ Mendonça, C Eiras, DS Soares, M José, JRS Leite (2013). Development and antibacterial activity of cashew gum-based silver nanoparticles. Intl J Mol Sci 14:4969-4981

Raffi M, F Hussain, T Bhatti, J Akhter, A Hameed, M Hasan (2008). Antibacterial characterization of silver nanoparticles against $E$. Coli ATCC-15224. J Mater Sci Technol 24:192-196

Ramanathan T, S Gurudeeban, K Satyavani, K Kathiresan, (2012). PP24: Pharmacological studies: Antimicrobial, antioxidant and antiaggregant activities of coastal she oak (Casuarina equisetifolia). In: Proceedings of International Conference on Environmental Security for Food and Health@, Vol 18, pp:6-18. Kanyakumari (ed.). February, 2012

Saranya V, SU Gowrie (2016). Green synthesis, optimization, characterization of silver nanoparticles using Casuarina equisetifolia bark extract and its assays. World J Pharm Res 3:797-814

Saratale RG, I Karuppusamy, GD Saratale, A Pugazhendhi, G Kumar, Y Park, GS Ghodake, RN Bharagava, JR Banu, HS Shin (2018). A comprehensive review on green nanomaterials using biological systems: Recent perception and their future applications. Colloids Surf. B. Biointerfaces 170:20-35

Singh C, J Kumar, P Kumar, BS Chauhan, KN Tiwari, SK Mishra, S Srikrishna, R Saini, G Nath, J Singh (2019). Green synthesis of silver nanoparticles using aqueous leaf extract of Premna integrifolia (L.) rich in polyphenols and evaluation of their antioxidant, antibacterial and cytotoxic activity. Biotechnol Biotechnol Equip 33:359-371

Thakkar KN, SS Mhatre, RY Parikh (2010). Biological synthesis of metallic nanoparticles. Nanomed Nanotechnol Biol Med 6:257-262

Wang X, L Zhang, C Ma, R Song, H Hou, D Li (2009). Enrichment and separation of silver from waste solutions by metal ion imprinted membrane. Hydrometallurgy 100:82-86

Yang Y, P Jin, X Zhang, N Ravichandran, H Ying, C Yu, H Ying, Y Xu, J Yin, K Wang (2017). New epigallocatechin gallate (egcg) nanocomplexes co-assembled with 3-mercapto-1-hexanol and $\beta$ lactoglobulin for improvement of antitumor activity. J Med Nano 13:805-814

Yoon WJ, KY Jung, J Liu, T Duraisamy, R Revur, FL Teixeira, S Sengupta, PR Berger (2010). Plasmon-enhanced optical absorption and photocurrent in organic bulk heterojunction photovoltaic devices using self-assembled layer of silver nanoparticles. Sol Ener Mater Sol Cells 94:128-132 